\title{
CELL FUSION AND SOMATIC CELL GENETICS ${ }^{1}$
}

\author{
(The Japan Society of Human Genetics Award Lecture) \\ Yoshio OKADA \\ Department of Animal Virology, Institute of Microbial \\ Diseases, Osaka University, Osaka, Japan
}

It is my great honour to have been chosen as this year's recipient of the Annual Prize awarded by the Japan Society of Human Genetics. More than two decades have passed since cell fusion was first discovered through HVJ. At that time, I felt that this phenomenon would be useful for cell genetics and biology. However, progress to date utilizing the phenomenon of cell fusion has far surpassed those initial expectations. I have been surprised at how much has been accomplished in a natural science by the development of only one technique. It is as if it changed the basic concept of the field. Somatic cell genetics and cell biology of Mammal suddenly emerged as a modern science when the phenomenon of cell fusion was established. It is truly wonderful to me that the virus HVJ (or Sendai virus) was isolated in Japan and that the phenomenon of cell fusion was discovered and established in this country also. However, the progress that has been made in this field since these findings has, of course, been due to the cooperation of many distinguished scientists from all parts of the world.

In classic genetics, an individual animal body is understood as only the phenotype of the genomes of a fertilized egg. According to this theory, a somatic cell is only one member of the whole body. However, after Carrel (1912) demonstrated the growth of cells from a tissue of chick embryo in vitro, it became possible to handle a somatic cell as an autonomous individual in a Petri dish. We know that a complete set of genomes derived from parents is included in each somatic cell and that a somatic cell at the level of genomes is equivalent to a fertilized egg. Thus, we can use a somatic cell in vitro for genetic analysis instead of a whole body. Unfortunately, however, technical freedom for genetic analysis of somatic cells in culture has not been so great because they do not show such phenomena as the male and female conjugation of germ cells. However, the development of artificial cell fusion solved the largest problem and led the way to the establishment of somatic cell genetics.

The first advance in somatic cell genetics was gene mapping on individual human chromosomes. Weiss et al. (1967) found the important fact in a hybrid of man

\footnotetext{
${ }^{1}$ Presented at the 23rd Annual Meeting of the Japan Society of Human Genetics at Niigata, Japan, October 29-30, 1978.
} 
and mouse that the chromosomal balance was unstable and that chromosomes derived from man disappeared gradually on serial passage of the hybrid progeny in culture. As a result, we can isolate hybrid clones containing mouse chromosomes as well as a human chromosome. The human phenotypes appearing on the hybrid depend on the genes of the human chromosome. Over the past 10 years, more than 100 human genes have been mapped by this technique, and one or more phenotype markers have been indicated on each human chromosome.

The next was an analysis of genetical control corresponding to luxury functions of somatic cells. We can modify the expression of some luxury functions by fusion or hybridization of a cell expressing a luxury function and another not expressing one. From this experiment, we have learned that 1) substances controlling gene expression are actually present and the amount is limited in a cell, and 2) the genecontrolling substances do not show species-specificity in function.

The third advance was in human hereditary diseases. A genetic complementary test is possible using fusion of somatic cells. It has been confirmed that some complementary groups, different in defective cistrons, are present in patients suffering from the same syndrome. For example, Xeroderma pigmentosum (XP) cells show a defect in the excision repair system of damaged DNA. By utilizing cell fusion by HVJ, five complementary groups have been identified in XP patients. This means that five or more genes participate in the excision repair system of man and that an XP child cannot result from a union of two XP patients providing they belong to differing groups.

The fourth advance was the development of a new technique for the introduction of macromolecules into living cells. Three such methods have been produced in our laboratory. Method $I$ is a technique utilizing diffusion of macromolecules through cell membranes, which occurs at an early stage of the cell fusion reaction. Method II utilizes the fusion of cell membranes with viral envelope vesicles, in which macromolecules have been trapped. Method III employs the fusion of cells with erythrocyte-ghosts, in which macromolecules have been trapped. We demonstrated that the defective function of XP cells was restored to normal by the introduction of endonuclease $V$ of T4 bacteriophage $(1975,1977)$, which was accomplished by Method I. The endonuclease V was effective in all five complement groups of cells. This means that the incision step of the excision repair system in man in controlled by at least five genes. Furthermore, the results suggest a possible method for clinical treatment of XP patients. 